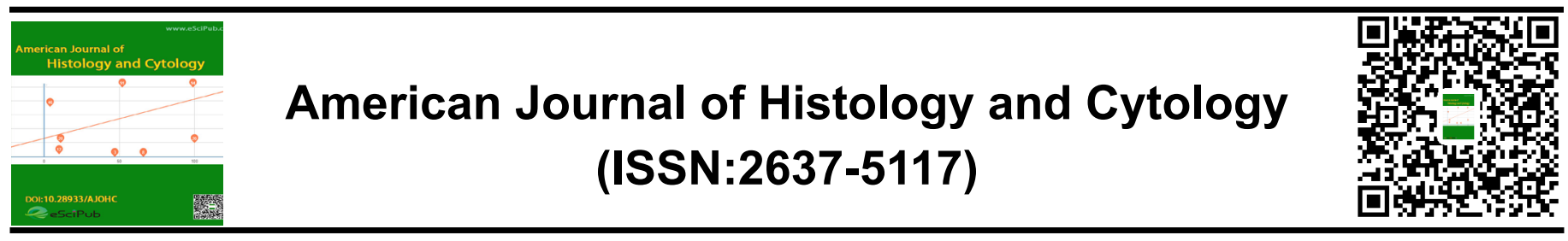

\title{
Pathological Approach to Pleural Malignant Mesothelioma
}

\author{
Vanessa Fonseca-Ferrer, MD; Luis Gerena-Montano, MD , Héctor A. Nieves Figueroa, MD; \\ William Rodriguez-Cintrón, MD
}

VA Caribbean Healthcare System, San Juan P.R; Pulmonary and Critical Care Medicine

\begin{abstract}
Exposure to asbestos can lead to asbestosis or malignancy 10-40 years after initial exposure $\left[^{1]}\right.$. Although its use has been banned in multiple countries, past occupational exposure leads to most cases that we see in present time. Malignant mesothelioma is an insidious and rare neoplasm that can arise from mesothelial surface cells, being Malignant Pleural Mesothelioma (MPM) the most common type. Lifetime risk of developing mesothelioma among asbestos workers can be as high as 10 percent and latency period is approximately $30-40$ years since time of exposure to development of disease ${ }^{[2]}$. Annual incidence in the united states is approximately 3,300 cases per year ${ }^{[3]}$. Median overall survival of patients with advanced unresectable disease is approximately 12 months $\left.{ }^{[4}\right]$. Clinical suspicion should arise in patients with previous exposure to asbestos who present with pleural thickening and/or effusion with associated respiratory symptoms. Most symptoms are nonspecific such as chest pain, dyspnea, cough and night sweats. Initial evaluation includes chest x-ray, contrast enhanced CT of the chest to find pleural abnormalities, thoracentesis and closed pleural biopsy. However, difficulties establishing diagnosis have been illustrated on studies where thoracentesis and pleural fluid cytology only yields diagnosis in $26 \%$ of cases. The diagnosis, then, is established by morphologic and immunohistochemistry findings of cytologic and *Correspondence to Author: Vanessa Fonseca-Ferrer, MD VA Caribbean Healthcare System, San Juan P.R; Pulmonary and Critical Care Medicine

How to cite this article:

Vanessa Fonseca-Ferrer, Luis Gerena-Montano, Héctor A. Nieves Figueroa, William Rodriguez-Cintrón. Pathological Approach to Pleural Malignant Mesothelioma. American Journal of Histology and Cytology, 2022, 5:14. surgical specimens.
\end{abstract}




\section{Case presentation:}

We present a 74-year-old African American male with a past medical history of hypertension, benign prostate hyperplasia and chronic asbestos work exposure. Patient worked as a handler of pipes for water distribution at St. Croix. He presented with worsening sharp pleuritic chest pain and shortness of breath on exertion. Denied cough, fever, hemoptysis, night sweats, recent travel, sick contacts, toxic habits, weight loss or recent travel. Physical examination was remarkable for left lung decreased breath sounds, and dull to percussion with decreased tactile fremitus. Chest $x$-ray showed evidence of large left pleural effusion with pleural thickening at the lung apex and peripheral nodular-pleural based opacity in the apex of the left lung (Figure 1). Chest CT with and without contrast showed evidence of large left pleural effusion and diffuse nodular thickening of the pleura with compressive atelectasis of the left lung and left to right cardio mediastinal shifting (Figure 2). PET CT demonstrated on coronal, sagittal and transverse projections increased FDG concentration throughout the whole left thoracic pleura that corresponded to pleural thickening segments of CT (Figure 3). Left pleural thoracentesis extracted $1200 \mathrm{ml}$ of unfixed turbid amber fluid with increased cell count as specified on table 1. Lights criteria positive for exudative effusion, as evidenced on table 2, and cultures with no bacterial growth. Cytopathology showed loose aggregates and clusters of malignant cells with irregular nuclei and reactive mesothelial cells. Immunohistochemistry showed tumoral cells strongly immunoreactive to CK7, Podoplanin, Calretinin, WT1 and Mesothelin (Figure 4). Immunohistochemical expression coupled with the radiologic impression compatible with the diagnosis of malignant mesothelioma. However, neoplasm unresectable reason why patient was started on systemic chemotherapy.

Table 1: Pleural effusion analysis cell count/differential

\begin{tabular}{|c|c|c|}
\hline Pleural fluid analysis & Results/Status & Reference range \\
\hline Fluid color & Amber & Pale yellow \\
\hline Fluid appearance & Turbid & Clear \\
\hline Supernatant color & Yellow & N/A \\
\hline Supernatant appearance & Clear & $0-1000$ \\
\hline Cell count, cm ${ }^{3}$ & 1,790 & $<25$ \\
\hline PMNs, \% & 3 & N/A \\
\hline LYMPHS, \% & 74 & N/A \\
\hline Macrophages, $\%$ & 2 & N/A \\
\hline Mesothelial, $\%$ & 1 & \\
\hline
\end{tabular}




\section{Discussion:}

Occupational asbestos exposure has decreased but remains a significant problem due previous exposure years before. Incidence of mesothelioma in the US has been in decline, secondary to asbestos exposure control [5]. Pleural involvement is a hallmark of asbestos exposure, characterized by pleural plaques and diffuse pleural thickening. These are benign findings due to asbestos exposure, however it is important to exclude malignancy in view of these findings. As part of initial evaluation of symptoms chest $x$-ray is the initial exam and then chest computed tomography is pursued. Most common radiographic findings include unilateral large pleural effusion, pleural mass or diffuse pleural thickening, pleural plaques and/or calcifications. When patient presents with symptomatic pleural effusion initial thoracentesis should be performed and pleural fluid cytologic examination should follow. Histologic examination should be supplemented by immunohistochemistry which includes selected markers of epithelioid mesothelioma (Caretinin, WT1, Podoplanin and Keratin 5/6) ${ }^{[6]}$. Once pleural mesothelioma is diagnosed, integrated positron emission tomography with computed tomography is obtained as part of staging assessment ${ }^{[7]}$. Only a minority of patients are candidates for surgical resection, reason why the mainstay of therapy is systemic chemotherapy.

\section{Conclusion:}

Malignant pleural mesothelioma remains the most common type of malignant mesotheliomas. One need to consider occupational exposure to asbestos specially in construction workers, pipefitters, plumbers, insulation workers, among others as they are the most at risk. Accuracy of detection and diagnosis of pleural malignant mesothelioma has improved with widespread use of immunohistochemistry. Imaging studies are needed for staging of tumor and although only a minority of cases are surgical candidates' systemic chemotherapy is available for therapy in these patients reason why early detection, especially in those with known previous occupational exposure, is important for early management.
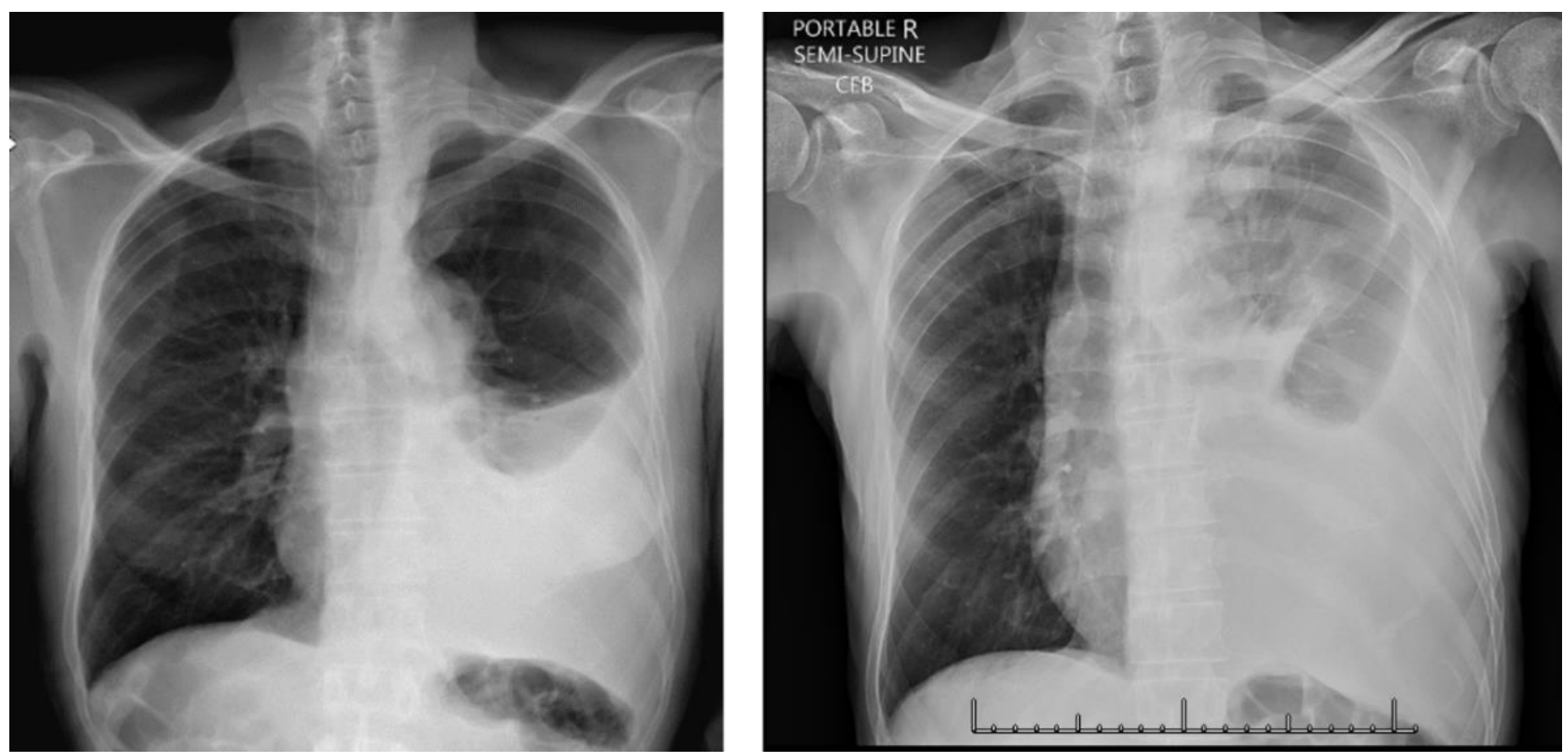

Figure 1: Chest $\mathrm{x}$-ray. Chest $\mathrm{x}$-ray showing large pleural effusion occupying most of left hemithorax and producing significant mass effect upon mediastinal structures. Right lung is well-expanded and grossly clear. 
Vanessa Fonseca-Ferrer et al., AJOHC, 2022, 5:14

Table 2: Pleural fluid chemical analysis

\begin{tabular}{|c|c|c|}
\hline Chemistry pleural fluid/serum & Result & Reference range \\
\hline Pleural $\mathrm{pH}$ & 7.48 & $\mathrm{~N} / \mathrm{A}$ \\
\hline Pleural total protein, g/dL & 5.6 & $\mathrm{~N} / \mathrm{A}$ \\
\hline Pleural LDH, U/L & 326 & $\mathrm{~N} / \mathrm{A}$ \\
\hline Pleural glucose, $\mathrm{mg} / \mathrm{dL}$ & 108 & $60-200$ \\
\hline Serum LDH, U/L & 178 & $6.0-8.5$ \\
\hline Serum total protein, g/dL & 8.6 & \\
\hline
\end{tabular}
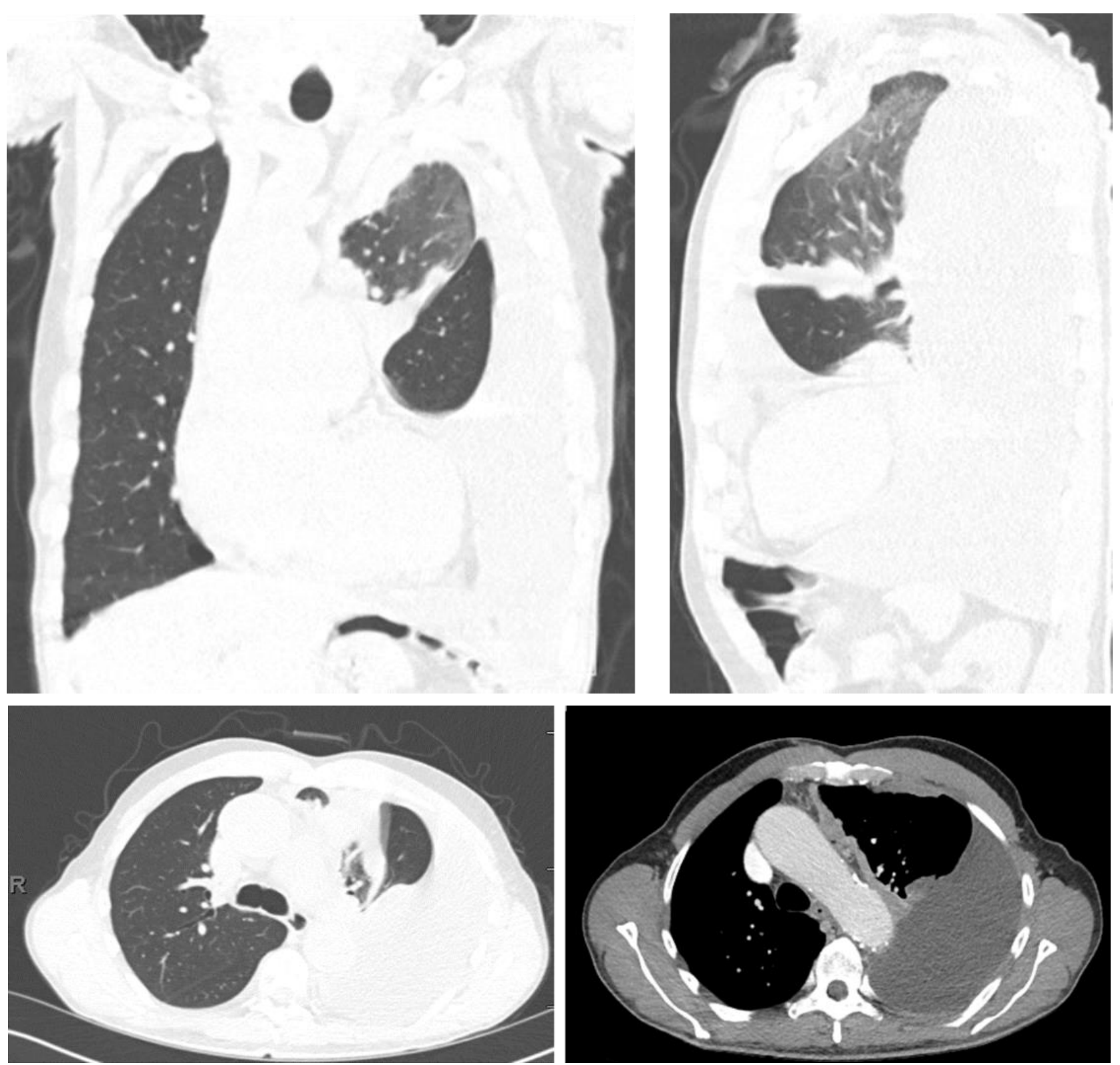

Figure 2: Computed tomography. Chest CT scan Coronal, sagittal and transverse projections showing diffuse nodular thickening and enhancement of the left pleura with associated large pleural effusion, compressive atelectasis of the left lung and left to right cardio mediastinal shifting. 

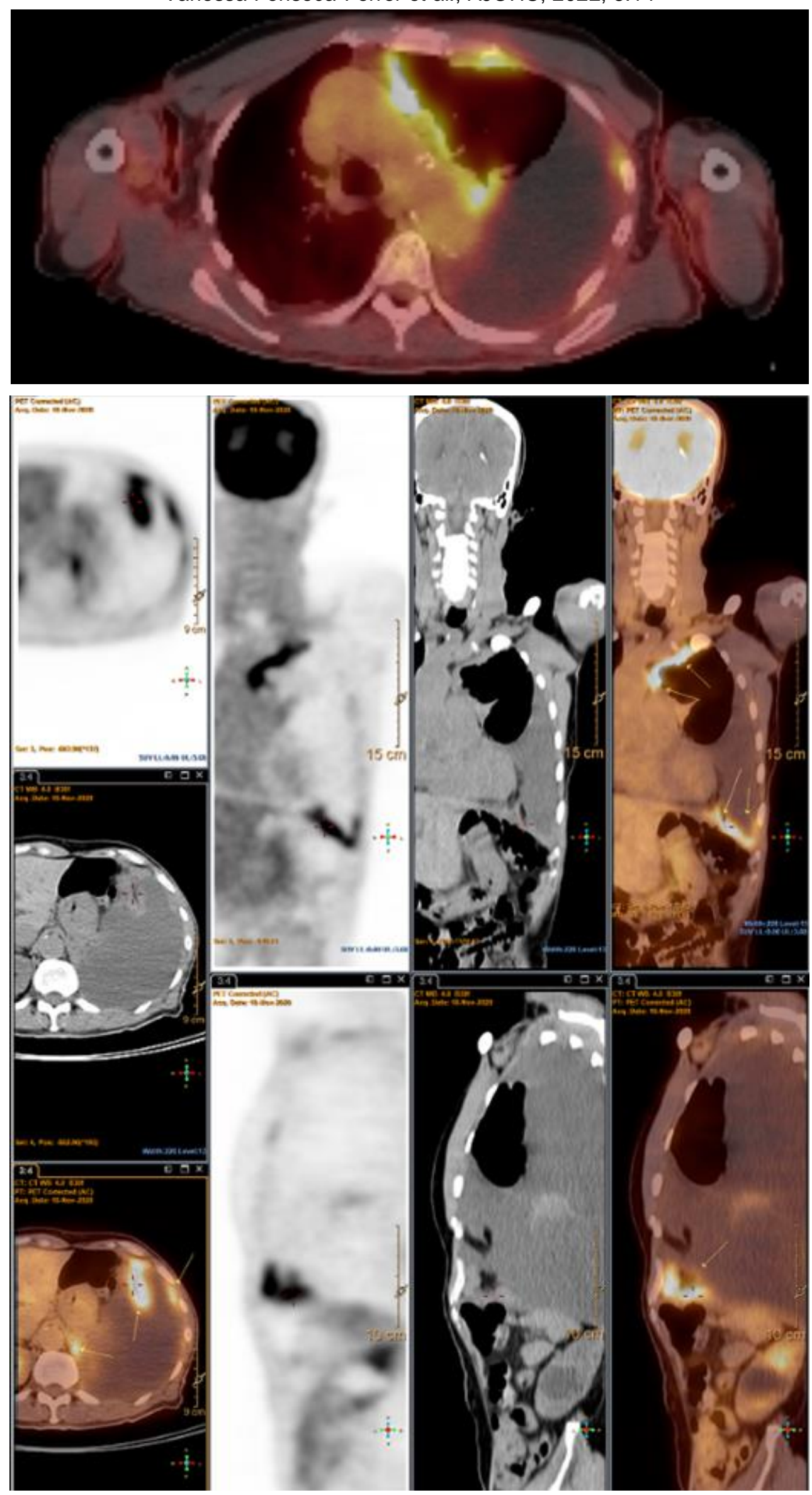

Figure 3: PET. PET CT Coronal, sagittal and transverse projections showing moderate diffused uneven increased FDG concentration throughout the whole left thoracic pleura corresponding on the CT scan to lobulated thickening segments of the pleura. 


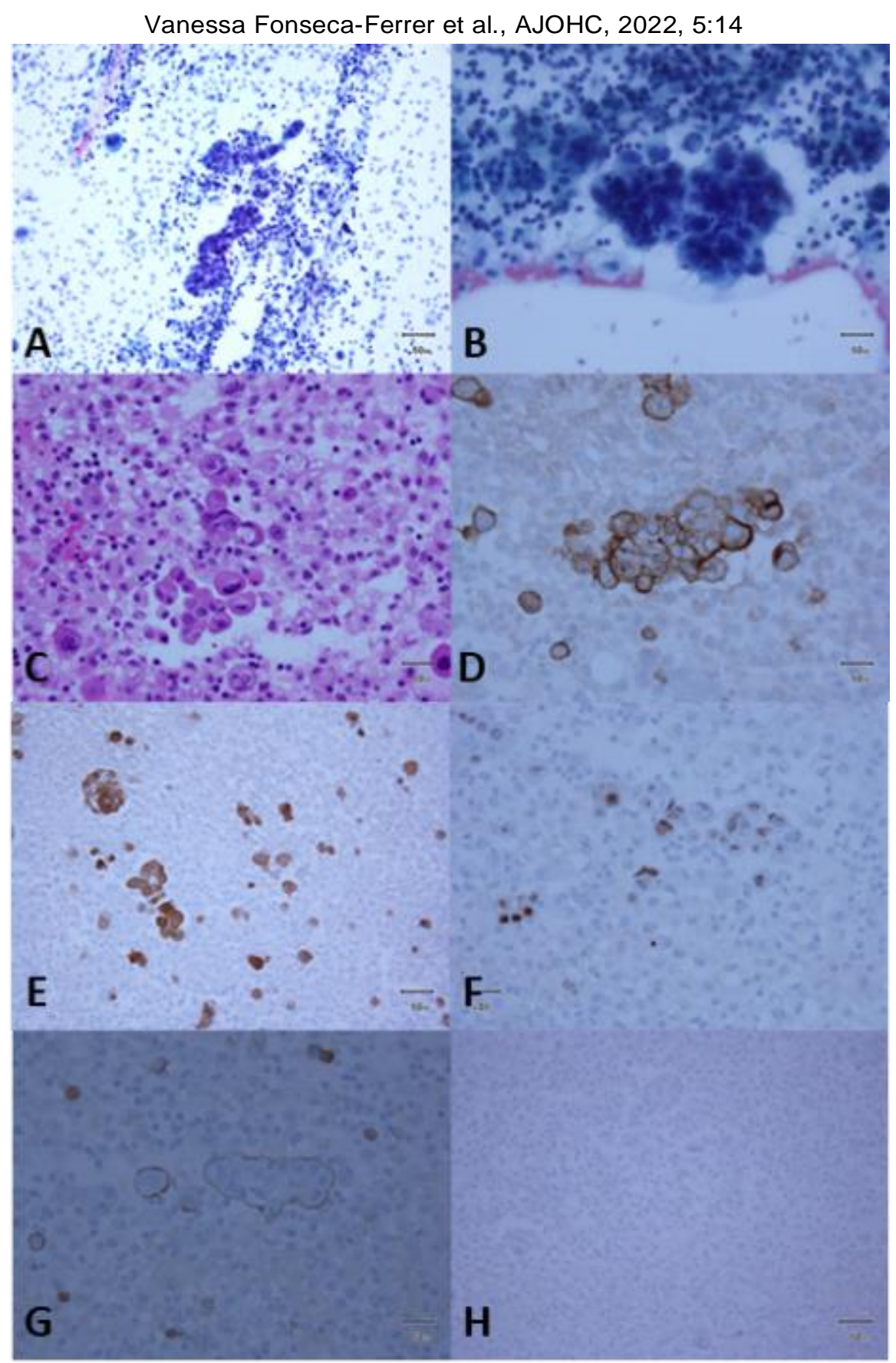

Figure 4: Pleural effusion immunohistochemistry. Immunohistochemistry A \& B: PAP smear, C: Cell block H\&E, D: Mesothelin, E: Calretinin, F: CK7, G: Podoplanin (D2-40), H: Non-reactive for Napsin-A, TTF-1, CK20 and P-53.

\section{References:}

[1]. Centers for Disease Control and Prevention (CDC). Asbestosis-related years of potential life lost before age 65 years--United States, 19682005. MMWR Morb Mortal Wkly Rep. 2008 Dec 12;57(49):1321-5. PMID: 19078920.

[2]. Selikoff IJ, Hammond EC, Seidman H. Latency of asbestos disease among insulation workers in the United States and Canada. Cancer. 1980 Dec 15;46(12):2736-40. doi: 10.1002/10970142(19801215)46:12<2736::aidcncr2820461233>3.0.c0;2-I. PMID: 7448712.
[3]. Teta MJ, Mink PJ, Lau E, Sceurman BK, Foster ED. US mesothelioma patterns 1973-2002: indicators of change and insights into background rates. Eur J Cancer Prev. 2008 Nov;17(6):525-34.

doi: 10.1097/CEJ.0b013e3282f0c0a2. PMID: 18941374.

[4]. Vogelzang NJ, Rusthoven JJ, Symanowski J, et al: Phase III study of pemetrexed in combination with cisplatin versus cisplatin alone in patients with malignant pleural mesothelioma. J Clin Oncol 21:2636-2644, 2003 
Vanessa Fonseca-Ferrer et al., AJOHC, 2022, 5:14

[5]. Price B. Analysis of current trends in United States mesothelioma incidence. Am J Epidemiol. 1997 Feb 1;145(3):211-8. doi: 10.1093/oxfordjournals.aje.a009093. PMID: 9012593.

[6]. Pathology and Genetics: Tumors of the Lung, Pleura, Thymus, and Heart, IARC, 2004.

[7]. Kindler HL, Ismaila N, Armato SG 3rd, Bueno R, Hesdorffer M, Jahan T, Jones CM, Miettinen M, Pass H, Rimner A, Rusch V, Sterman D, Thomas A, Hassan R. Treatment of Malignant Pleural Mesothelioma: American Society of Clinical Oncology Clinical Practice Guideline. J Clin Oncol. 2018 May 1;36(13):1343-1373. doi: 10.1200/JCO.2017.76.6394. Epub 2018 Jan 18. PMID: 29346042; PMCID: PMC8058628. 